\title{
Full Tensor Gradient of Simulated Gravity Data for Prospect Scale Delineation
}

\author{
Hendra Grandis* \& Darharta Dahrin \\ Applied Geophysics Research Group, Faculty of Mining and Petroleum Engineering, \\ Institut Teknologi Bandung, Jalan Ganesha 10, Bandung 40132, Indonesia \\ *Email: grandis@geoph.itb.ac.id
}

\begin{abstract}
Gravity gradiometry measurement allows imaging of anomalous sources in more detail than conventional gravity data. The availability of this new technique is limited to airborne gravity surveys using very specific instrumentation. In principle, the gravity gradients can be calculated from the vertical component of the gravity commonly measured in a ground-based gravity survey. We present a calculation of the full tensor gradient (FTG) of the gravity employing the Fourier transformation. The calculation was applied to synthetic data associated with a simple block model and also with a more realistic model. The latter corresponds to a 3D model in which a thin coal layer is embedded in a sedimentary environment. Our results show the utility of the FTG of the gravity for prospect scale delineation.
\end{abstract}

Keywords: anomaly enhancement; fast Fourier transform (FFT); filtering; gravity; potential fields methods.

\section{$1 \quad$ Introduction}

In gravity and magnetic surveys, the spatial (i.e. horizontal and vertical) gradients of potential field data may enhance subtle prospect scale anomalies directly related to the anomalous sources. Recent advances in instrumentation for gravity data acquisition allow direct measurement of the gradients of all gravity vector components $\left(g_{x}, g_{y}\right.$ and $\left.g_{z}\right)$ along all coordinate directions $x, y$, and $z$. However, measurement of the so-called full tensor gradient (FTG) of the gravity is generally performed on airborne platforms $[1,2]$. This tends to limit the application of FTG gravity surveys to large exploration campaigns with considerable budgets, i.e. for oil and gas prospecting in difficult tectonic settings $[3,4]$.

In this paper the term "full tensor gradient of the gravity" refers to the complete tensor elements of the gravity gradient, although they are obtained from calculation, not from direct measurement. Furthermore, the complete tensor elements of the gravity gradient are calculated only from the vertical component of the gravity commonly measured in the field (i.e. ground-based survey). In the Fourier domain, the gravity gradient is equal to the multiplication of the gravity

Received October $11^{\text {th }}, 2013$, Revised February $20^{\text {th }} 2014$, Accepted for publication May $12^{\text {th }}, 2014$.

Copyright (C) 2014 Published by ITB Journal Publisher, ISSN: 2337-5760, DOI: 10.5614/j.math.fund.sci.2014.46.2.1 
with the wave number along the direction of the derivative [5]. Using the 2D FFT technique, we calculated the FTG from the vertical gravity of a simple 3D block model. The results gave comparable values (with low misfit) to the gradient tensor from direct calculation using a 3D forward modeling algorithm [6]. We also applied the method to enhance simulated gravity data associated with a thin coal layer embedded in a sedimentary environment, with satisfactory results.

\section{Full Tensor Gradient of Gravity}

The Full Tensor Gradient (FTG) of the gravity contains first derivatives of all gravity vector components $\mathbf{g}=\left[g_{x}, g_{y}, g_{z}\right]$ with respect to the $x$-, $y$ - and $z$ directions in a Cartesian coordinate system, as given by,

$$
\underline{\Delta \mathbf{g}}=\left[\Delta g_{i j}\right]=\left[\begin{array}{ccc}
\frac{\partial g_{x}}{\partial x} & \frac{\partial g_{x}}{\partial y} & \frac{\partial g_{x}}{\partial z} \\
\frac{\partial g_{y}}{\partial x} & \frac{\partial g_{y}}{\partial y} & \frac{\partial g_{y}}{\partial z} \\
\frac{\partial g_{z}}{\partial x} & \frac{\partial g_{z}}{\partial y} & \frac{\partial g_{z}}{\partial z}
\end{array}\right] ; i, j=x, y \text { or } z .
$$

The gravitational acceleration or gravity is a potential gradient $(\mathbf{g}=-\nabla \phi)$ such that the tensor components in Eq. (1) are in fact second derivatives of the potential $\phi$. Therefore, there is symmetry of the tensor components, i.e. $G_{x y}=G_{y x}, G_{x z}=G_{z x}$ and $G_{y z}=G_{z y}$ giving only 6 independent tensor components, i.e. $G_{x x}, G_{x y}, G_{x z}, G_{y y}, G_{y z}$ and $G_{z z}$.

The gravitational potential $\phi$ follows Laplace's equation $\nabla^{2} \phi=0$, such that $\nabla \times \mathbf{g}$ $=0$. Then, by considering the symmetry of the tensor components and the derivative property of the Fourier transform, we have the following Fourier transform pairs [5],

$$
\begin{aligned}
& \frac{\partial g_{z}}{\partial y}=\frac{\partial g_{y}}{\partial z} \Leftrightarrow i k_{y} G_{z}=|\mathbf{k}| G_{y}, \\
& \frac{\partial g_{x}}{\partial z}=\frac{\partial g_{z}}{\partial x} \Leftrightarrow|\mathbf{k}| G_{x}=i k_{x} G_{z},
\end{aligned}
$$




$$
\frac{\partial g_{y}}{\partial x}=\frac{\partial g_{x}}{\partial y} \Leftrightarrow i k_{x} G_{y}=i k_{y} G_{x},
$$

where $G_{x}\left(k_{x}, k_{y}\right), G_{y}\left(k_{x}, k_{y}\right)$ and $G_{z}\left(k_{x}, k_{y}\right)$ are 2D Fourier transformed gravity components of $g_{x}, g_{y}$ and $g_{z}$ respectively, while $k_{x}$ and $k_{y}$ are wave numbers with the following property [5],

$$
|\mathbf{k}|=\sqrt{\left(k_{x}^{2}+k_{y}^{2}\right)}=i k_{z}
$$

In conventional gravity surveys, the only quantity measured is the vertical component, i.e. $g_{z}$. However, the relationships of the horizontal components $g_{x}$ and $g_{y}$ with the vertical component $g_{z}$ of the gravity in the Fourier domain can be obtained from Eq. (2) such that,

$$
\begin{aligned}
& g_{x} \Leftrightarrow G_{x}=\frac{i k_{x}}{|\mathbf{k}|} G_{z}, \\
& g_{y} \Leftrightarrow G_{y}=\frac{i k_{y}}{|\mathbf{k}|} G_{z} .
\end{aligned}
$$

Therefore, the components of the gravity gradient tensor can be theoretically calculated from the measured gravity anomaly by using the following equation,

$$
\underline{\Delta \mathbf{g}}=\left[\Delta g_{i j}\right]=F^{-1}\left(\left[\begin{array}{ccc}
\frac{-k_{x}^{2}}{|\mathbf{k}|} & \frac{-k_{x} k_{y}}{|\mathbf{k}|} & i k_{x} \\
\frac{-k_{x} k_{y}}{|\mathbf{k}|} & \frac{-k_{y}^{2}}{|\mathbf{k}|} & i k_{y} \\
i k_{x} & i k_{y} & |\mathbf{k}|
\end{array}\right] G_{z}(\mathbf{k})\right) \text {, }
$$

where $F^{-1}$ is the inverse Fourier transformation and $|\mathbf{k}| \square 0$. Eq. (5) shows that the gradient calculation can be performed in the Fourier domain as the multiplication of an input signal with a filter function. In addition to anomaly enhancement, gradient maps will exhibit amplified high-frequency components (including noise) since the filter function is proportional to the spatial frequency or wave number. 


\section{Simple Block Model}

We performed a 3D gravity forward modeling to obtain the theoretical gravity response of a simple block model represented by a symmetrical vertical prism with constant density contrast relative to its surrounding environment. The practical formula for calculating the gravitational attraction of a vertical rectangular prism has previously been proposed by several authors $[7,8]$. The vertical component of the gravity $\left(g_{z}\right)$ at an observation point at the origin $O(0$, $0,0)$ due to a vertical prism with a unit density is given by [9],

$$
\begin{gathered}
g_{z}=-G\left[\left[\left[x \operatorname { l n } \left(y+\left(x^{2}+y^{2}+z^{2}\right)^{1 / 2}+y \ln \left(x+\left(x^{2}+y^{2}+z^{2}\right)^{1 / 2}\right.\right.\right.\right.\right. \\
\left.\left.+z \arctan \left(z\left(x^{2}+y^{2}+z^{2}\right)^{1 / 2} x^{-1} y^{-1}\right]_{x 1}^{x 2}\right]_{y 1}^{y 2}\right]_{z 1}^{z 2}
\end{gathered}
$$

where $\mathrm{G}$ in this case is the gravitational constant. With edges parallel to the reference axes, the prism is defined by the minimum and maximum values of the coordinate interval formed by $\left[x_{1}, x_{2}\right],\left[y_{1}, y_{2}\right]$ and $\left[z_{1}, z_{2}\right]$ in $x$-, $y$-, and $z$ directions respectively.

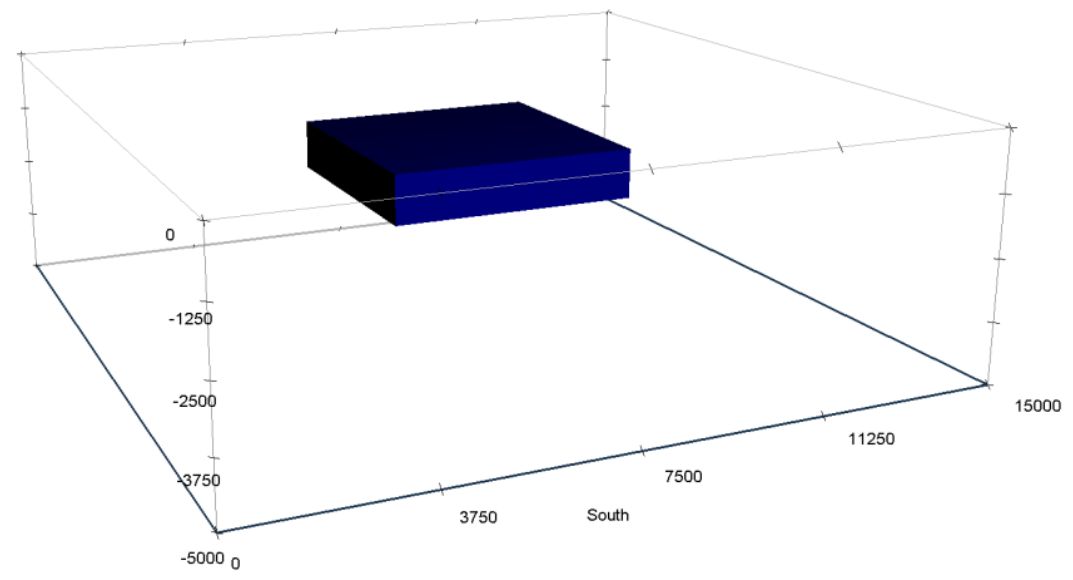

Figure 1 A perspective view of a simple block model measuring $5 \times 5 \times 1 \mathrm{~km}^{3}$ at $1 \mathrm{~km}$ depth with a density contrast of $0.5 \mathrm{gram} / \mathrm{cm}^{3}$.

In simulating the gravity gradient tensor, several authors have used different geometrical dimensions for the synthetic model in accordance with the order of magnitude of their target, i.e. from superficial [e.g. 10] to regional-scale [e.g. 11] anomalous sources. Nevertheless, the gradiometer data are almost linearly 
$\Delta g_{x x}$
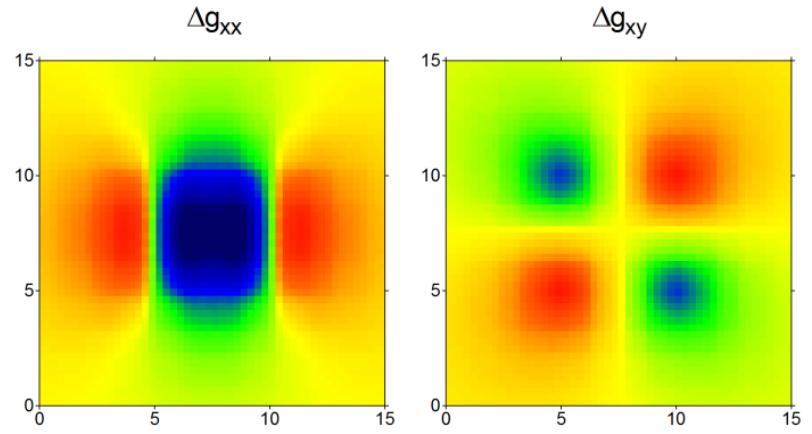

$\Delta g_{\mathrm{yy}}$

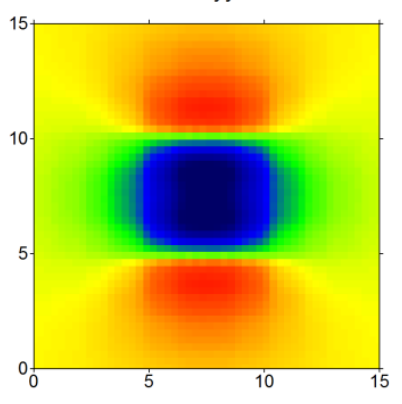

$g_{z}$

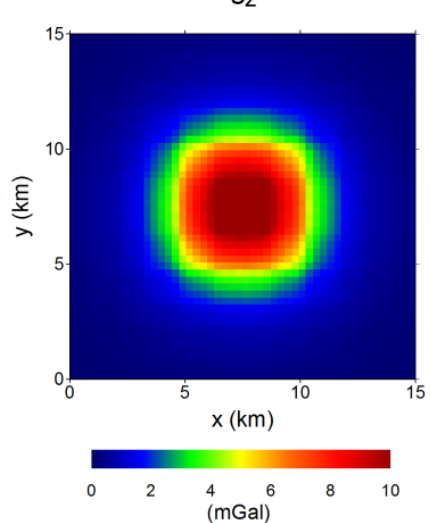

$\Delta g_{x z}$

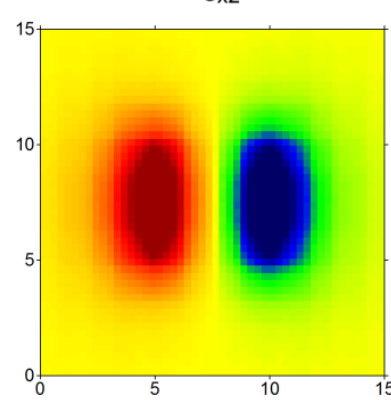

$\Delta g_{\mathrm{yz}}$

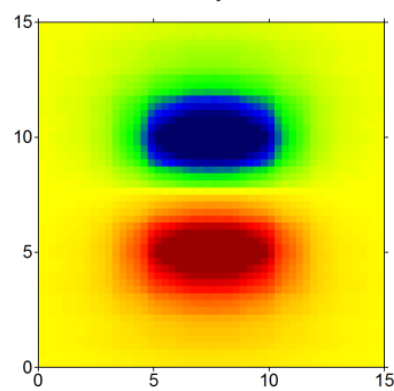

$\Delta g_{\mathrm{zz}}$

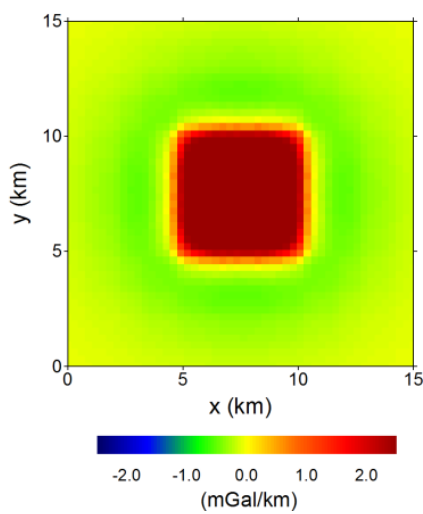

Figure 2 Full tensor gradient of the gravity (independent components only) obtained from forward modeling calculation. All components use the same color scale shown for $\Delta g_{z z}$ (lower right panel). The vertical gravity response of a simple 3D block model is shown at the lower left panel. 

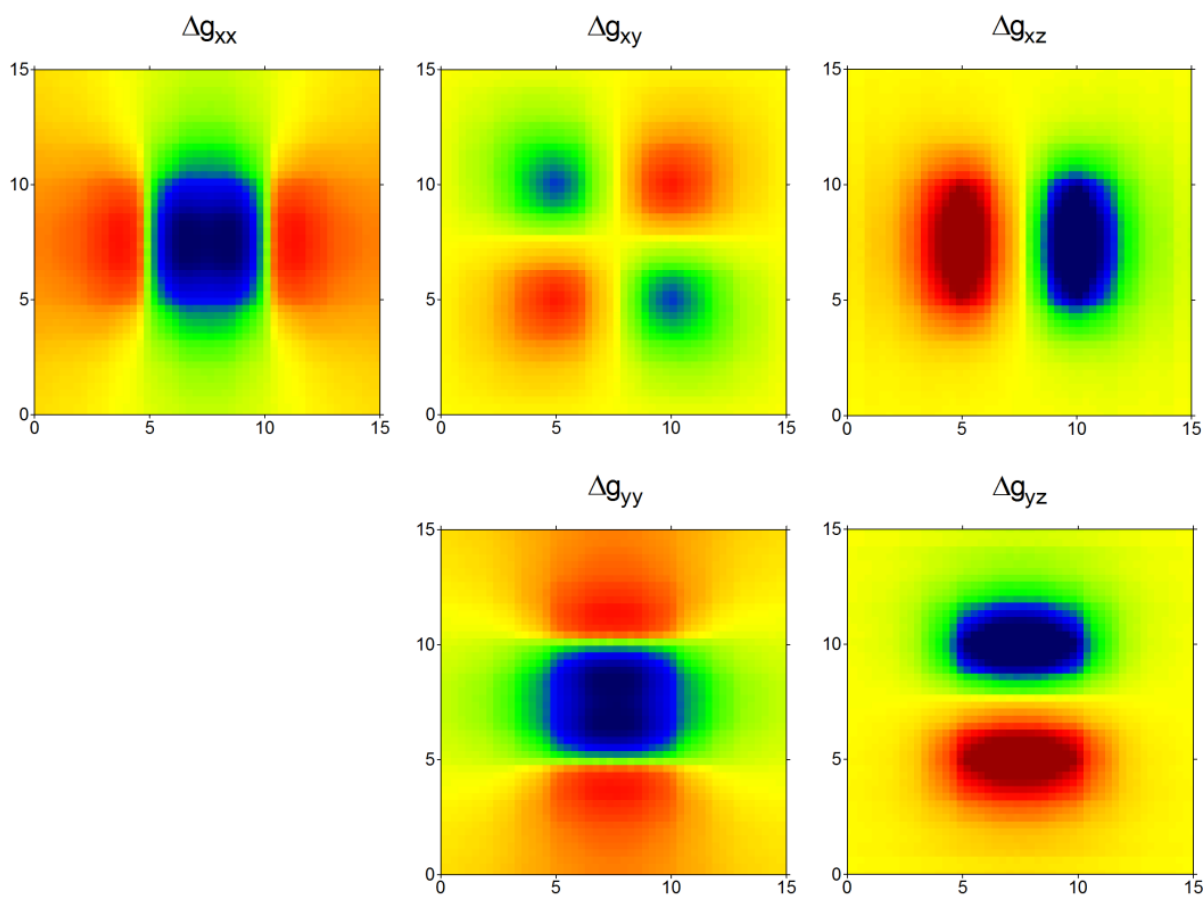

$g_{z}$
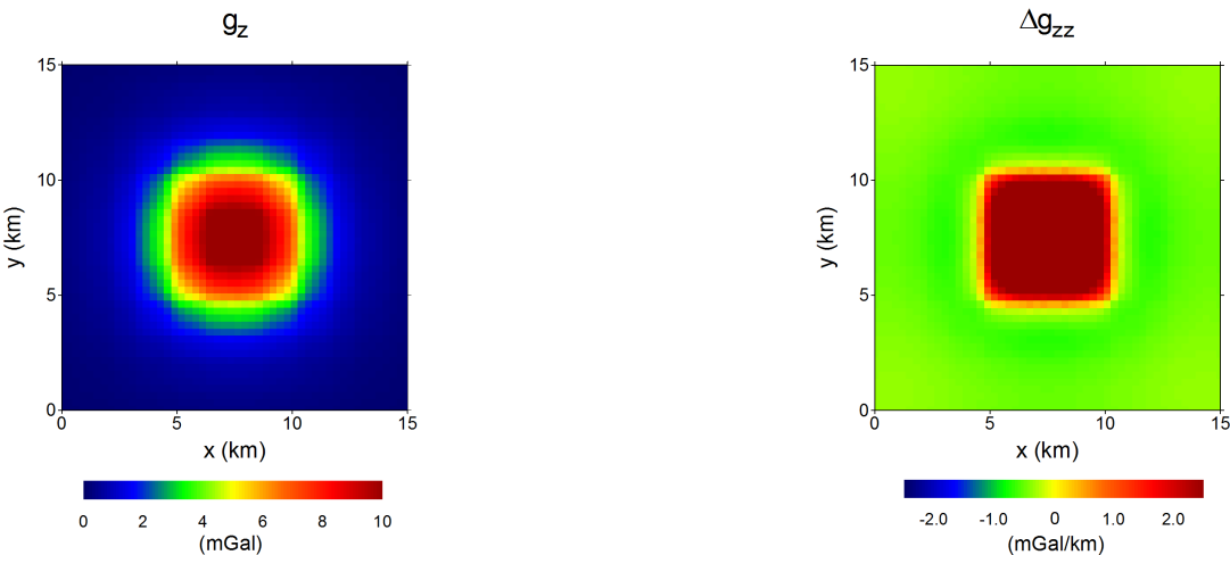

Figure 3 Full tensor gradient of the gravity (independent components only) obtained from FFT calculation with input from vertical gravity response of a simple 3D block model shown in the lower left panel (noise-free synthetic data). All components use the same color scale as for $\Delta g_{z z}$ (lower right panel). 
scalable and independent of the length units [11]. In order to obtain spatially contrasting results in the gradient calculation, we used a block model with $5 \times 5$ $\times 1 \mathrm{~km}^{3}$ in $x$-, $y$-, and $z$-directions, located at $1 \mathrm{~km}$ depth. The prism with a density contrast of $0.5 \mathrm{gram} / \mathrm{cm}^{3}$ was positioned at the center of an area of 15 by $15 \mathrm{~km}^{2}$ and the calculation of the theoretical gravity anomaly was done for 0.3 by $0.3 \mathrm{~km}$ grid cells (Figure 1). Normally distributed (Gaussian) noise with zero mean and 5\% standard deviation was then added to the model response to simulate real gravity data. The FTG of the gravity was calculated by using the 2D FFT technique [9]. As a comparison, we also calculated the gravity gradient tensor components of a vertical prism model by using an algorithm proposed by Montana, et al. [6], the results of which are presented in Figure 2 in the form of maps for each independent tensor component.

The gradient tensor components from the noise-free and the noise-added synthetic data are shown in Figure 3 and Figure 4 respectively. The magnitude of the gradient tensor components is relatively small and they span different intervals from one component to the other. However, for comparison purposes, we have used the same colour scale from -2.5 to $+2.5 \mathrm{mGal} / \mathrm{km}(1 \mathrm{mGal} / \mathrm{km}=$ 10 Eötvös) for all tensor components $\left(\left[\Delta g_{i j}\right]\right)$ and from 0 to $10 \mathrm{mGal}$ for the vertical component of the gravity $\left(g_{z}\right)$ in Figures 2,3 and 4 . The actual interval for each component of the noise-free gravity gradient tensor is presented in Table 1 to the nearest tenth of $\mathrm{mGal} / \mathrm{km}$. In general, the smallest magnitude is the gradient along two perpendicular planar directions $\left(\Delta g_{x y}\right)$, while the largest is the derivative along horizontal and vertical directions $\left(\Delta g_{x z}\right.$ and $\left.\Delta g_{y z}\right)$. In addition, lateral boundaries are mostly enhanced in the gradient containing components perpendicular to those boundaries $\left(\Delta g_{x x}\right.$ and $\left.\Delta g_{y y}\right)$ and also in the vertical gradient $\left(\Delta g_{z z}\right)$.

Table 1 Interval values for each component of the noise-free FTG.

\begin{tabular}{ccc}
\hline Component & Minimum (mGal/km) & Maximum (mGal/km) \\
\hline$g_{x x}$ & -2.6 & +1.4 \\
$g_{x y}$ & -1.5 & +1.5 \\
$g_{x z}$ & -3.9 & +3.9 \\
$g_{y y}$ & -2.6 & +1.4 \\
$g_{y z}$ & -3.9 & +3.9 \\
$g_{z z}$ & -0.5 & +5.1 \\
\hline
\end{tabular}

Qualitatively, there is no obvious difference between the FTG of the gravity from FFT calculation (Figure 3) or from 3D forward modeling (Figure 2). This comparison validates the approximation of the gravity gradient calculated from observed gravity data $\left(g_{z}\right)$. The tensor components of the gravity gradient 
calculated from noise-added synthetic data (Figure 4) still retain the global pattern of the corresponding components derived from the 3D forward modeling presented in Figure 2.
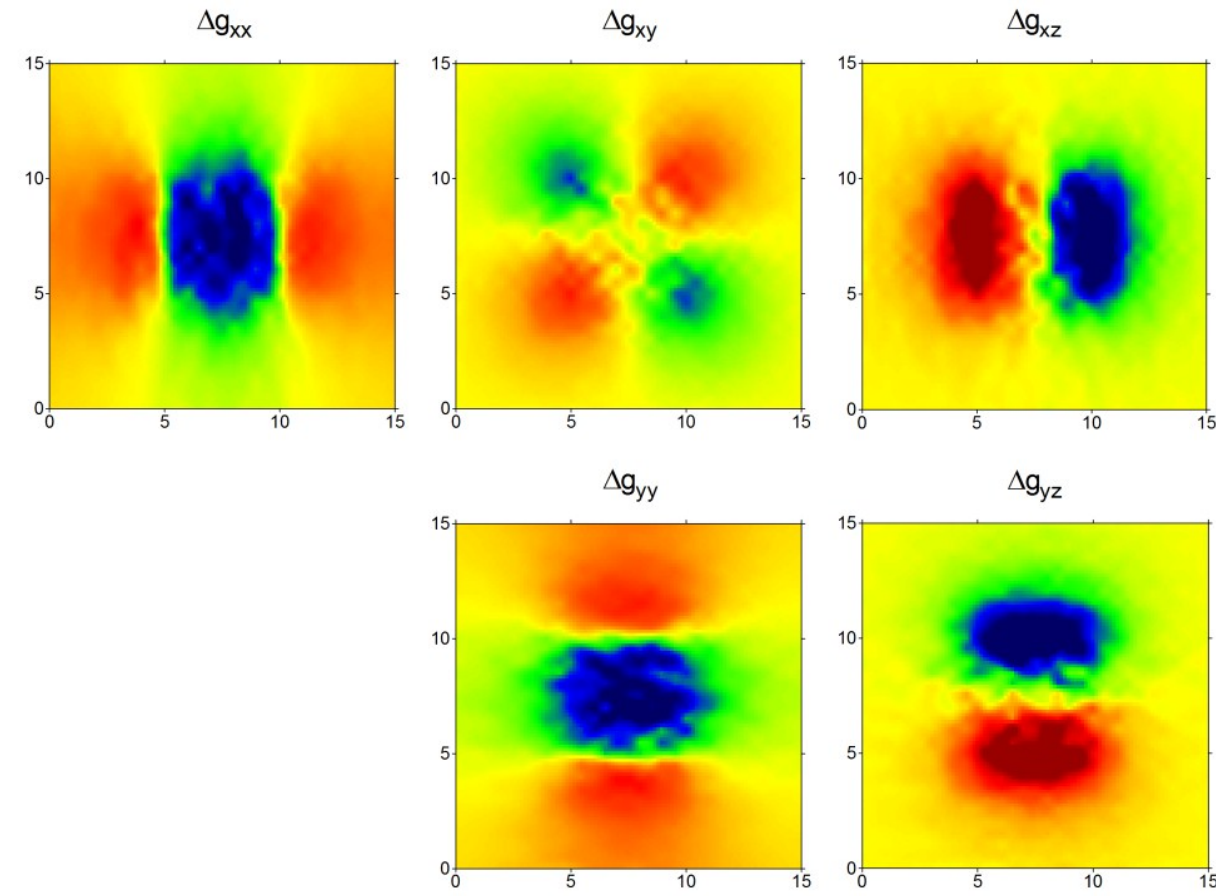

$g_{z}$
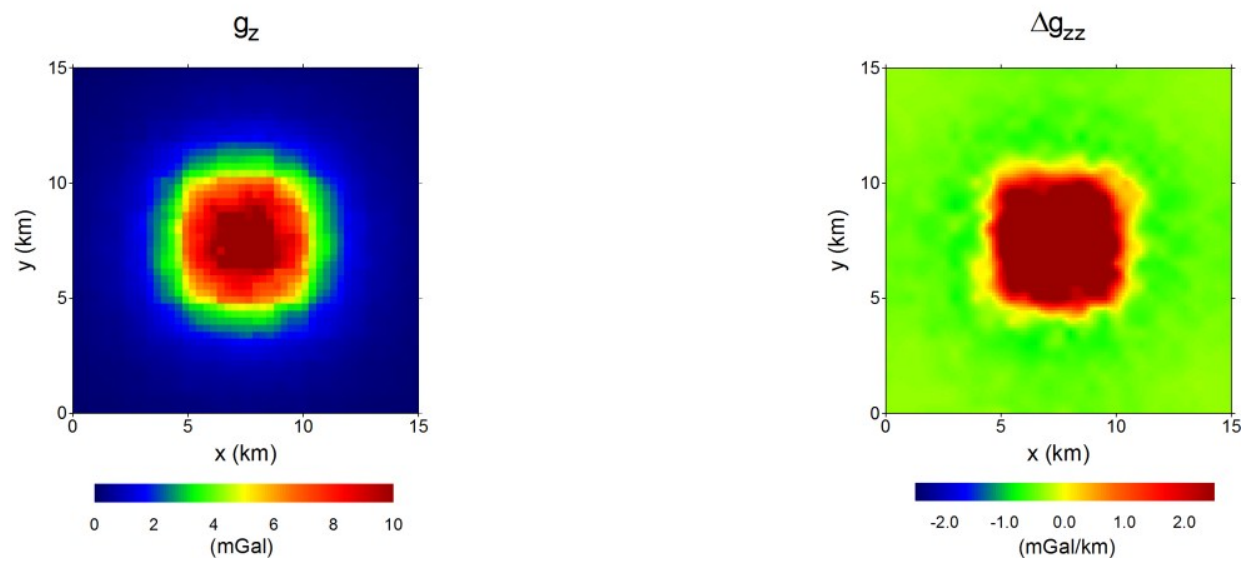

Figure 4 Full tensor gradient of the gravity (independent components only) obtained from FFT calculation with input from vertical gravity response of a simple 3D block model shown at the lower left panel (synthetic data with 5\% gaussian noise). All components use the same color scale as for $\Delta g_{z z}$ (lower right panel). 
Table 2 presents the absolute root mean square (RMS) errors of the tensor components from both the noise-free and the noise-added synthetic data compared to the result from the 3D forward modeling. The average errors for all components were 0.100 and $0.529 \mathrm{mGal} / \mathrm{km}$ for the noise-free and the noiseadded synthetic data respectively. Because of such error amplification (approximately 5-fold) due to noise addition (5\%) in the synthetic data, calculated gradients from real or field gravity data having higher noise content must be interpreted cautiously.

Table 2 RMS error in $\mathrm{mGal} / \mathrm{km}$.

\begin{tabular}{ccc}
\hline Component & Noise Free & $\mathbf{5 \%}$ Noise \\
\hline$g_{x x}$ & 0.139 & 0.466 \\
$g_{x y}$ & 0.059 & 0.307 \\
$g_{x z}$ & 0.014 & 0.520 \\
$g_{y y}$ & 0.139 & 0.496 \\
$g_{y z}$ & 0.014 & 0.553 \\
$g_{z z}$ & 0.236 & 0.831 \\
\hline average & $\mathbf{0 . 1 0 0}$ & $\mathbf{0 . 5 2 9}$ \\
\hline
\end{tabular}

\section{Simulated Gravity Data}

We simulated gravity data associated with a more realistic 3D model in which a thin coal layer is embedded in a regional sedimentary basin. We used available seismic data from an area with a typical coal-bed methane (CBM) prospect to construct the sedimentary layers. The exact location of the area is not relevant, therefore the coordinates of all subsequent maps were set as arbitrary for an area of 50 by $50 \mathrm{~km}^{2}$. Figure 5 shows the interpreted horizons from the seismic data along a South-West to North-East profile. The sedimentary layers overlying the basement are simply identified as layer-1 to layer-5 with densities estimated from well data.

Isolated coal spots were added between layer-2 and layer-3 to represent a CBM bearing formation. Within layer-3, coal spots with different thicknesses (i.e. 20, 50 and 100 meter) are located approximately at a 500 to 800 meter depth interval. The top of the coal layer coincides with the bottom of layer-2 (see Figure 5). A density of $1.7 \mathrm{gram} / \mathrm{cm}^{3}$ was assumed for the coal layer, such that the density contrast with its surrounding, i.e. layer-3 $\left(2.16 \mathrm{gram} / \mathrm{cm}^{3}\right)$, is -0.46 $\operatorname{gram} / \mathrm{cm}^{3}$. 

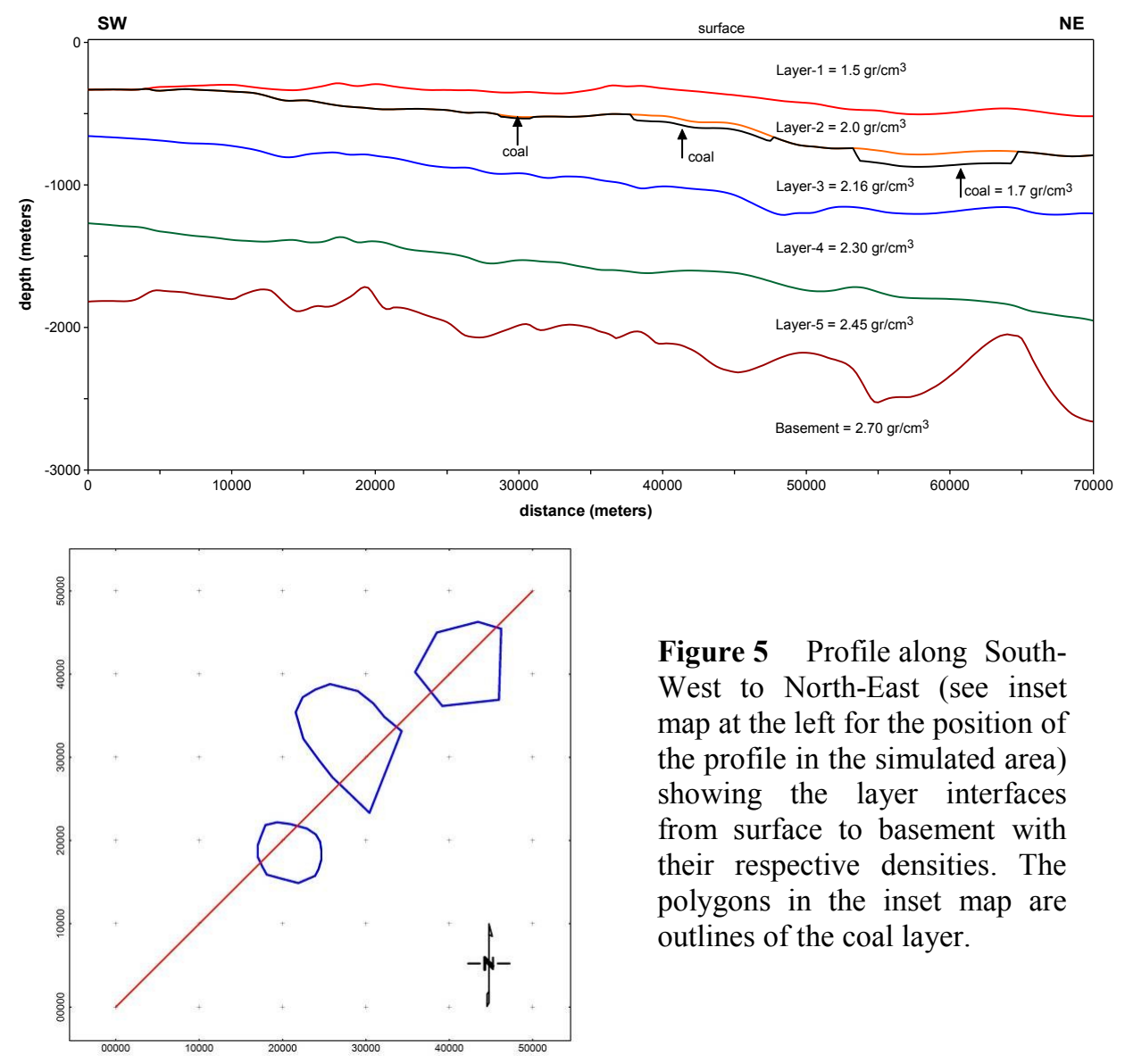

Figure 5 Profile along SouthWest to North-East (see inset map at the left for the position of the profile in the simulated area) showing the layer interfaces from surface to basement with their respective densities. The polygons in the inset map are outlines of the coal layer.

In 3D gravity forward modeling, a more complex model is usually decomposed into many small prisms with uniform size. The gravity response of such a model is the superposition of contribution from each unitary prism expressed in Eq. (6). For our case it was simpler to use Parker's formula $[9,12]$ to calculate the gravity anomaly caused by an uneven, uniform layer of material by means of a series of Fourier transforms. The relationship between vertical gravity effect and its causative mass topography $h(\mathbf{r})$ in the Fourier domain is given by $[12,13]$,

$$
F(\Delta g)=-2 \pi G \rho \exp \left(-|\mathbf{k}| z_{0}\right) \sum_{n=1}^{\infty} \frac{|\mathbf{k}|^{n-1}}{n !} F\left(h^{n}(\mathbf{r})\right)
$$

where $\mathbf{r}$ denotes position in the $x-y$ plane, $\mathbf{k}$ is the 2D wave number, $\rho$ is the density, and $G$ is the universal gravitational constant. Eq. (7) is convergent in 
the whole k-plane for $z_{0}>0$, where $z_{0}$ is the mean depth of the layer or density interface. The Fourier transformation has to be performed in 2D since the data are also $2 \mathrm{D}$, i.e. $h(\mathbf{r})$ is the depth of the density interface (positive downwards) as a function of the position $(x, y)$. Eq. (7) provides an efficient way to calculate the gravity effect caused by a subsurface mass anomaly or topography. Furthermore, it can be easily applied to multi-layer cases as well.

For the forward modeling calculation, horizons of sedimentary layers and basement were digitized with a 0.25 by $0.25 \mathrm{~km}^{2}$ grid interval. The gravity response calculated for the same grid is presented in Figure 6 . The gravity anomaly maps in Figure 6 represent the basin model without coal layer and the same model with a coal layer. We can observe the North-South direction of the regional trend with the lower anomaly at the East reflecting the deepening of the basement. Having almost the same range in gravity anomaly (from 438.7 to $469.7 \mathrm{mGal}$ ), the difference between these two gravity anomaly maps is hardly noticeable. This is related to the fact that the effect of the coal layer is very small due to its geometry (very thin with limited lateral extent) and to the low density contrast $\left(-0.46\right.$ gram $\left./ \mathrm{cm}^{3}\right)$.

The gravity gradient tensor for the simulated data (shown in Figure 7 only for $\Delta g_{x x}$ and $\Delta g_{z z}$ ) exhibits very complicated patterns so that it is difficult to identify the anomalous source from the components of the gradient tensor. Most of the components do not reflect the anomalous source, i.e. the coal layer. Difficulties in identifying anomalies from the gradient tensor of simulated data may arise from the interference between adjacent anomalous sources and the amplification of high frequency components of the anomalies.

The gravity gradient tensor might be valuable only for prospect scale, hence it is more appropriate for delineating more isolated gravity anomalies [14]. Therefore, we focused on the gradient tensor components associated with the thickest coal layer. We can observe the similarity, at least in the pattern, of the gradient tensor components between the simulated data and the ones from the simple anomaly (Figure 8 and Figure 9). In these figures the color scale for each component was set to cover the minimum and maximum values of the gradient (see the color scale in Figure 7 and Figure 2 for the simulated coal layer and the simple block model respectively). We can compare Figure 8 and Figure 9 to Figure 2, note that the simple block model has a positive density contrast while the coal has a negative density contrast such that the polarity of gradients is reversed. 

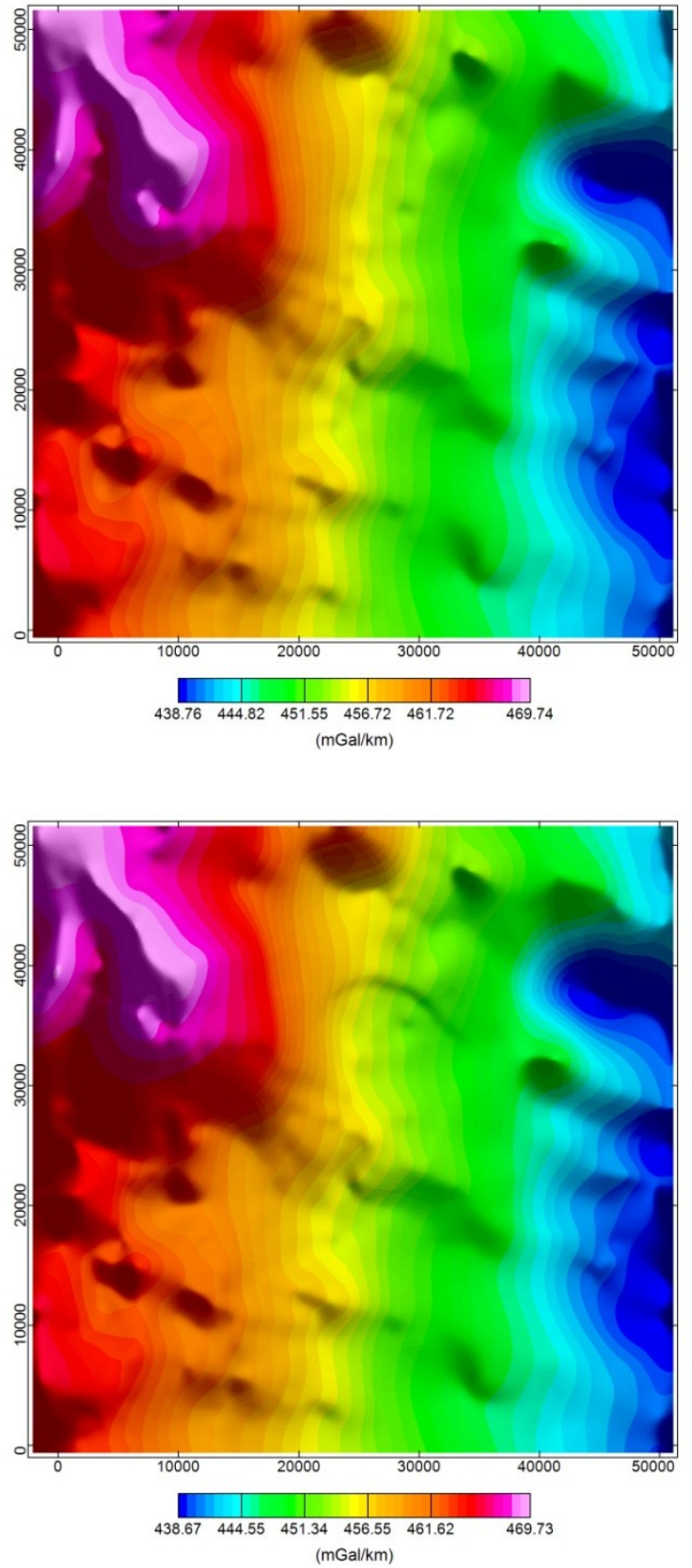

Figure 6 Simulated gravity anomaly maps associated with the basin model without coal layer (top) and the same model with coal layer (bottom). 

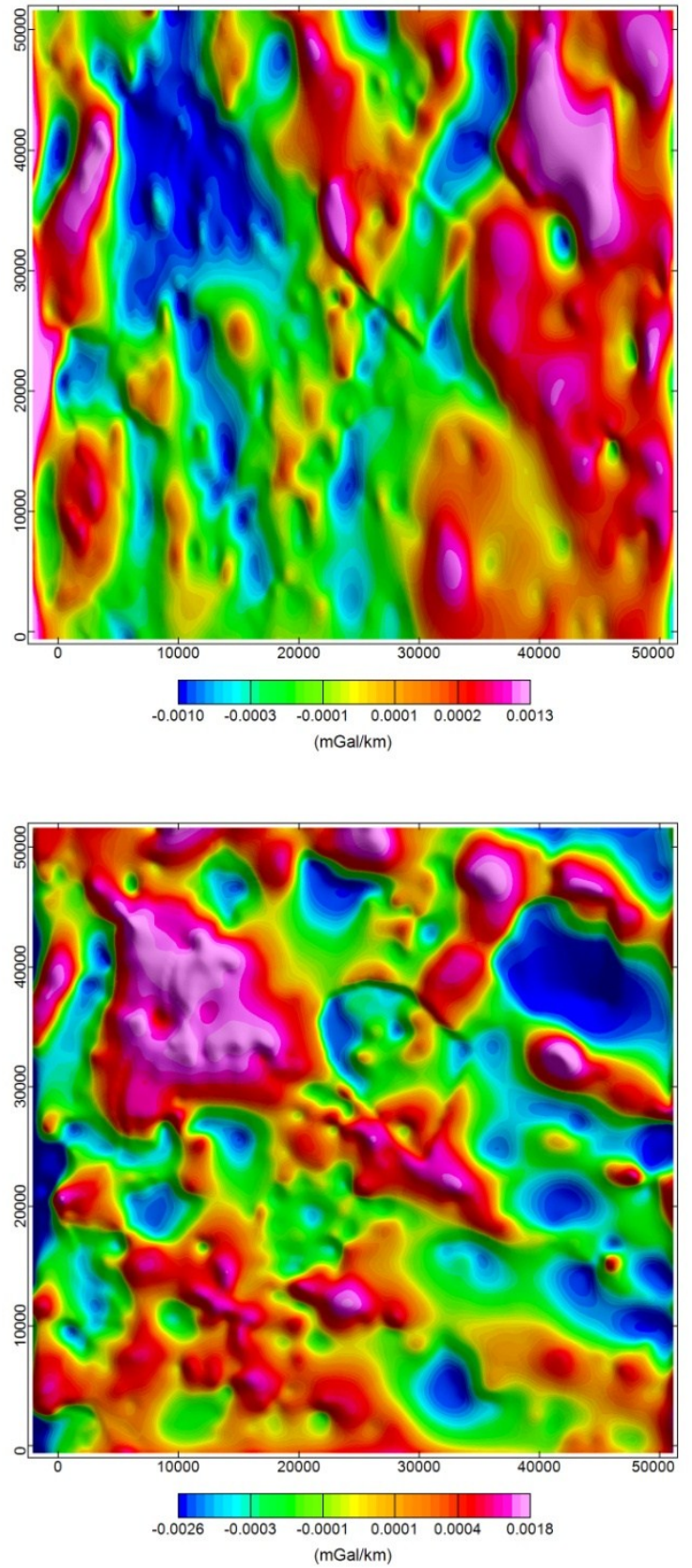

Figure 7 Gradient tensor components associated with the simulated gravity data, $\Delta g_{x x}$ (top) and $\Delta g_{z z}$ (bottom). 
$\Delta g_{x x}$
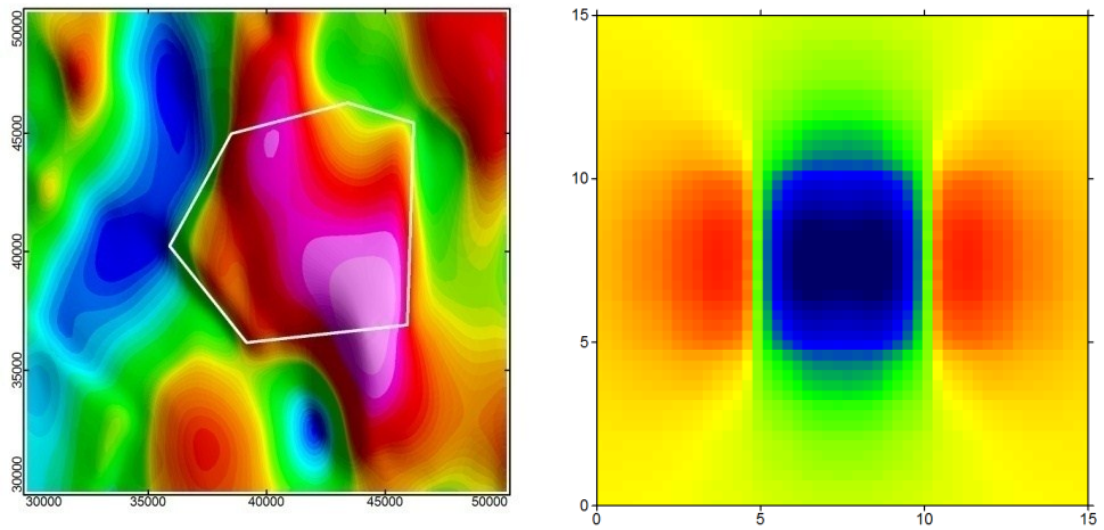

$\Delta g_{x y}$
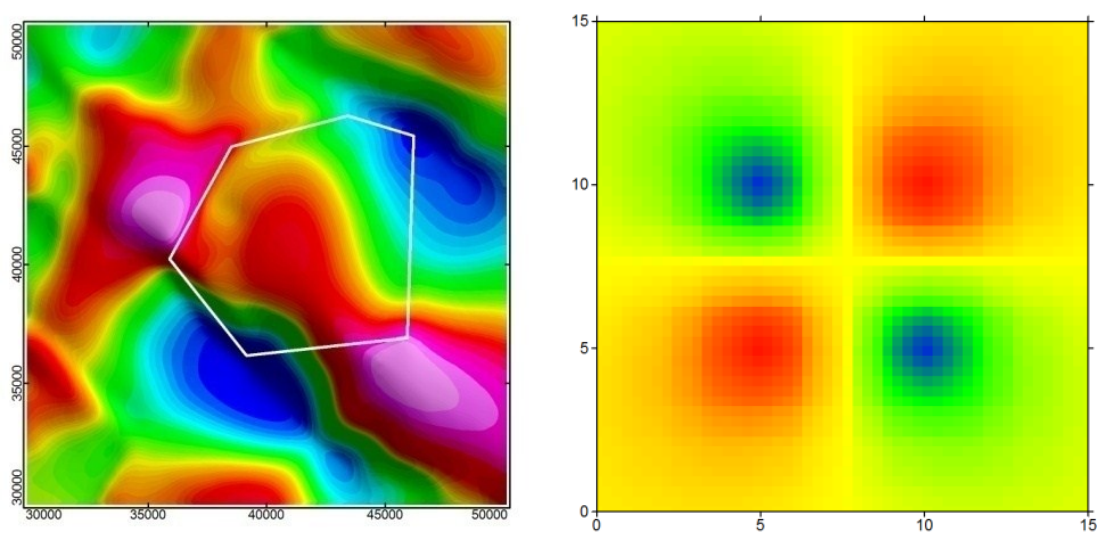

$\Delta g_{x z}$
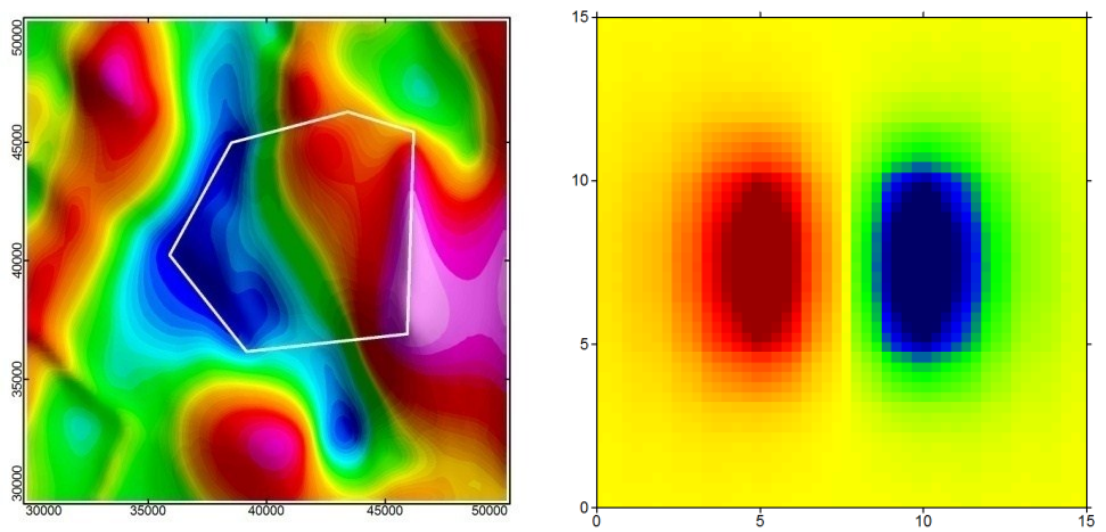

Figure 8 Independent components of the gravity gradient tensor $\left(\Delta g_{x x}, \Delta g_{x y}\right.$ and $\Delta g_{x z}$ ) of the simulated gravity data around the thickest coal layer (left) compared to the same components from Figure 2 (right). 
$\Delta g_{y y}$
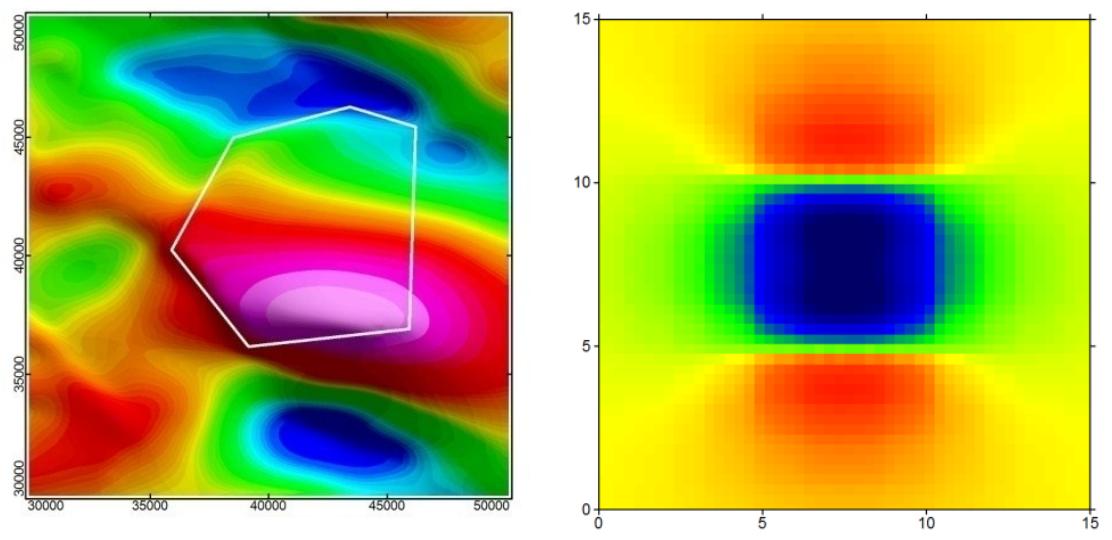

$\Delta g_{y z}$
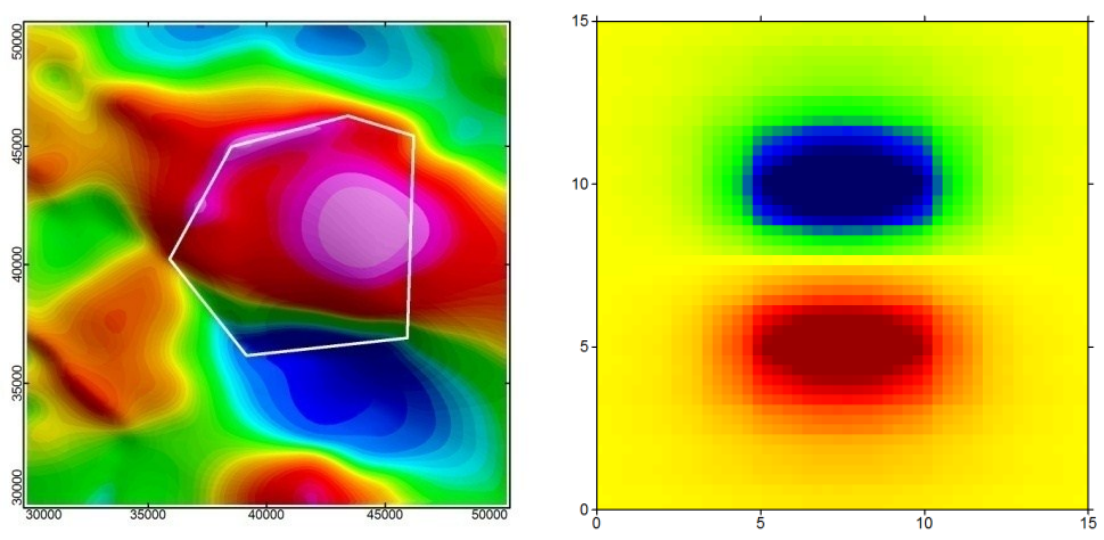

$\Delta g_{z z}$
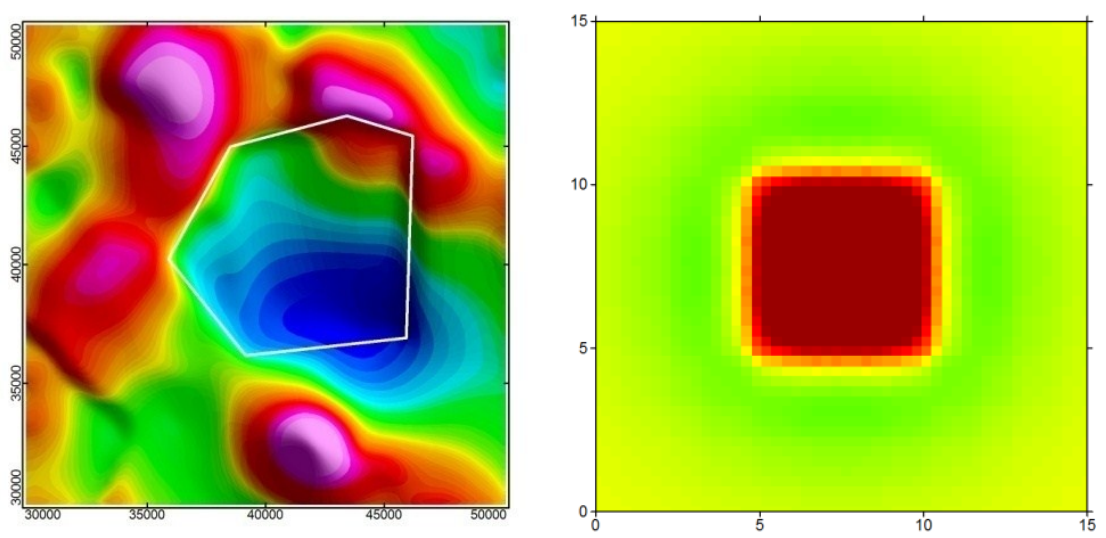

Figure 9 Independent components of the gravity gradient tensor ( $\Delta g_{y y}, \Delta g_{y z}$ and $\Delta g_{z z}$ ) of the simulated gravity data around the thickest coal layer (left) compared to the same components from Figure 2 (right). 


\section{Discussion}

It is obvious that the full tensor gradient (FTG) of the gravity contains more information about the anomalous source than conventional gravity data, i.e. vertical component $\left(g_{z}\right)$ only. In addition, it has interpolative power, which can be exploited to adapt the survey design as the exploration program progresses and also to increase the effective resolution of the data $[15,16]$. Furthermore, the modeling of gravity data involving their gradients for quantitative interpretation will result in more sharp lateral boundaries. It is also expected that the use of gradients in a gravity inversion will reduce the non-uniqueness inherent in the modeling of the potential fields data $[17,18]$.

In absence of actual measurement of the FTG, the gravity gradients can be calculated from the vertical component of the gravity by using the 2D FFT algorithm $[9,19]$. More information can be extracted from the calculated gravity gradients as they enhance the anomalous sources, more particularly their lateral boundaries. However, calculation of gradients in the Fourier domain also enhances high frequency components and noise present in the data. The latter necessitates precaution in the qualitative interpretation of the gradient maps.

Our simulations using a simple block model and a more realistic case also showed that the FTG of the gravity is most appropriate for prospect scale anomaly delineation. Therefore, the prospect scale or local anomaly must be identified first by applying conventional regional-residual anomaly separation based on the spatial frequency content of the anomaly. In the case described in this paper, the anomaly caused by the coal layer will certainly have a localized spatial extent relative to a wider anomaly due to the sedimentary environment. A suite of filtering techniques is available for this purpose [e.g. 20,21].

\section{Conclusion}

The use of the full tensor gradient (FTG) of the gravity for prospect scale anomaly delineation has been presented. In this case, the FTG of the gravity was calculated by using the 2D FFT algorithm from the vertical component of the gravity $\left(g_{z}\right)$. The algorithm was tested by calculating the gradient tensor of a simple 3D block model and a more realistic 3D model involving thin coal layers in a sedimentary environment. Detailed observation of independent components of the gradient tensor shows that the gradients of the isolated anomaly were similar in pattern to the gradients of the simple block model.

The results presented in this paper are part of our ongoing research. Future developments will include the inversion of gravity gradient data to obtain inverse models with less ambiguity and sharper boundaries of anomalies. 
Gravity and magnetic data share similar characteristics, since both are potentialfield data. Therefore, the techniques involving gradient tensor data are also applicable to magnetic data.

\section{References}

[1] Bell, R.E., Anderson, R.N. \& Pratson, L.F., Gravity Gradiometry Resurfaces, The Leading Edge, 16(1), pp. 55-59, 1997.

[2] Hansen, R.O., Pearson, W.C., de Ridder, E. \& Johnson, W.M., The Gravity Gradiometer: Basic Concepts and Trade Offs, The Leading Edge, 18(4), pp. 478-480, 1999.

[3] Oruç, B., Sertçelik, I., Kafadar, Ö. \& Selim, H.H., Structural Interpretation of the Erzurum Basin, Eastern Turkey, Using Curvature Gravity Gradient Tensor and Gravity Inversion of Basement Relief, Journal of Applied Geophysics, 88(1), pp. 105-113, 2013.

[4] Badmus, B.S., Sotona, N.K. \& Krieger, M., Gravity Support for Hydrocarbon Exploration at the Prospect Level, Journal of Emerging Trends in Engineering and Applied Sciences (JETEAS), 2(1), pp.1-6, 2011.

[5] Mickus, K.L. \& Hinojosa, J.H., The Complete Gravity Gradient Tensor derived from the Vertical Componentof Gravity: A Fourier Transform Technique, Journal of Applied Geophysics, 46(3), pp. 156-176, 2001.

[6] Montana, C.J., Mickus, K.L. \& Peeples, W.J., Program to Calculate the Gravitational Field and Gravity Gradient Tensor Resulting from a System of Right Rectangular Prisms, Computers \& Geosciences, 18(5), pp. 587-602, 1992.

[7] Nagy, D., Gravitational Attraction of a Right Rectangular Prism, Geophysics, 31(2), pp. 362-371, 1966.

[8] Plouff, D., Derivation of Formulas and FORTRAN Programs to Compute Gravity Anomalies of Prisms, National Technical Information Service No. PB-243-526, U.S. Department of Commerce, 1975.

[9] Blakely, R.J., Potential Theory in Gravity and Magnetic Applications, Cambridge University Press, 1996.

[10] Beiki, M., Analytic Signals of Gravity Gradient Tensorand Their Application to Estimate Source Location, Geophysics, 75(6), pp. I59-I74, 2010.

[11] Pilkington, M., Evaluating the Utility of Gravity Gradient Tensor Components, Geophysics, 79(1), pp. G1-G14, 2014.

[12] Parker, R.L., The Rapid Calculation of Potential Anomalies, Geophysical Journal of Royal Astronomical Society, 31(4), pp. 447-455, 1973. 
[13] Shin, Y.H., Choi, K.S. \& Xu, H., Three-Dimensional Forward and Inverse Models for Gravity Fields based on the Fast Fourier Transform, Computers \& Geosciences, 32(6), pp. 727-738, 2006.

[14] Murphy, C.A. \& Brewster, J., Target Delineation Using Full Tensor Gravity Gradiometry Data, ASEG Extented abstracts, 2007(1), pp. 1-3, 2007.

[15] Barns, G. \& Lumley, J., Processing Gravity Gradient Data, Geophysics, 76(2), pp. I33-I47, 2011.

[16] Barns, G., Interpolating The Gravity Field Using Full Tensor Gradient Measurements, First Break, 30(4), pp. 97-101, 2012.

[17] Grandis, H. \& Dahrin, D., Constrained Two-Dimensional Inversion of Gravity Data, Journal of Mathematical and Fundamental Sciences, 46(1), pp. 1-13, 2014.

[18] Pilkington, M., Analysis of Gravity Gradiometer Inverse Problems Using Optimal Design Measures, Geophysics, 77(2), pp. G25-G31, 2012.

[19] Buttkus, B., Spectral Analysis and Filter Theory in Applied Geophysics, Springer-Verlag, 2000.

[20] Grandis, H., Simulation of 3D Gravity Anomaly of Thin Coal Layer in Sedimentary Environment and its Delineation, International Journal of Tomography and Simulation, 26(2), pp. 1-11, 2014.

[21] Keating, P. \& Pinet, N., Use of Non-Linear Filtering for the RegionalResidual Separation of Potential Field Data, Journal of Applied Geophysics, 73(4), pp. 315-322, 2011. 